\title{
Findings of Cognitive Impairment at High Altitude: Relationships to Acetazolamide Use and Acute Mountain Sickness.
}

\author{
Lara Phillips \\ Thomas Jefferson University \\ Buddha Basnyat \\ Oxford University Clinical Research Unit - Nepal \\ Yuchiao Chang \\ Harvard Medical School \\ Erik R. Swenson \\ University of Washington \\ N. Stuart Harris \\ Harvard Medical School \\ Follow this and additional works at: https://jdc.jefferson.edu/emfp \\ Part of the Emergency Medicine Commons \\ Let us know how access to this document benefits you
}

\section{Recommended Citation}

Phillips, Lara; Basnyat, Buddha; Chang, Yuchiao; Swenson, Erik R.; and Harris, N. Stuart, "Findings of Cognitive Impairment at High Altitude: Relationships to Acetazolamide Use and Acute Mountain Sickness." (2017). Department of Emergency Medicine Faculty Papers. Paper 60. https://jdc.jefferson.edu/emfp/60

This Article is brought to you for free and open access by the Jefferson Digital Commons. The Jefferson Digital Commons is a service of Thomas Jefferson University's Center for Teaching and Learning (CTL). The Commons is a showcase for Jefferson books and journals, peer-reviewed scholarly publications, unique historical collections from the University archives, and teaching tools. The Jefferson Digital Commons allows researchers and interested readers anywhere in the world to learn about and keep up to date with Jefferson scholarship. This article has been accepted for inclusion in Department of Emergency Medicine Faculty Papers by an authorized administrator of the Jefferson Digital Commons. For more information, please contact: JeffersonDigitalCommons@jefferson.edu. 
Acute Mountain Sickness and Cognition acetazolamide use and acute mountain sickness

6 Lara Phillips, MD ${ }^{1,2}$, Buddha Basnyat $\mathrm{MD}^{3}$, Yuchiao Chang PhD ${ }^{1}$, Erik R. Swenson MD and N. Stuart Harris MD MFA ${ }^{1}$

$8{ }^{1}$ Division of Wilderness Medicine, Department of Emergency Medicine, Massachusetts

9 General Hospital, Harvard Medical School, Boston, MA 02114, USA.

$10{ }^{2}$ Department of Emergency Medicine, Thomas Jefferson University Hospital, Philadelphia, PA 19107, USA.

$12{ }^{3}$ Oxford University Clinical Research Unit - Nepal; Himalayan Rescue Association, Kathmandu, Nepal.

$14{ }^{4}$ Medical Service, Veterans Affairs Puget Sound Health Care System, University of Washington, Seattle, WA 98108, USA. 
Acute Mountain Sickness and Cognition

\section{Abstract}

2 Objective: Acute mountain sickness (AMS) is defined by patient-reported symptoms

3 using the Lake Louise Score (LLS), which does not necessarily provide insight into

4 possible central nervous system (CNS) dysfunction. Our hypothesis was that AMS might

5 be associated with cognitive impairment (CI) and may go undetected unless a sensitive

6 test is applied. A standardized test for mild CI could provide a potential new tool to better

7 characterize altitude-related CNS dysfunction.

8 Methods: We compared a cognitive screening tool with the LLS. We recruited adult

9 native English-speaking subjects visiting Himalayan Rescue Association aid posts in

10 Nepal at 3520m (11,550ft) and 4550m (14,930ft). Subjects were administered the LLS

11 and a slightly modified version of the Quick Mild Cognitive Impairment Screen (eQmci).

12 Medication use for altitude illness was recorded. LLS and eQmci scores were compared

13 using the Spearman correlation coefficient. A cut-off of $\geq 3$ with at least 1 point for

14 headache was used for the LLS to diagnose AMS and 67 or less for the eQmci to

15 diagnose CI. Data also included medication use.

16 Results: Seventy-nine subjects were enrolled. Twenty-two (28\%) subjects met criteria for

17 AMS and $17(22 \%)$ subjects met criteria for CI. There was a weak correlation $\left(\mathrm{r}^{2}=0.06\right.$,

$18 \mathrm{p}=0.04)$ between eQmci score and LLS. In matched subjects with identical LLS, recent

19 acetazolamide use was significantly associated with more frequent CI.

20 Conclusion: Field assessment of CI using a rapid standardized tool demonstrated a

21 substantial number of subjects had mild CI following rapid ascent to $3520-4550 \mathrm{~m}$

$22(11,548-14,927 \mathrm{ft})$. The weak correlation between the LLS and eQmci suggests that AMS 
Acute Mountain Sickness and Cognition

1 is not associated with CI. Use of acetazolamide may have an association with CI at all

2 levels of AMS severity.

3

4

5

6

7

8

9

10

11

12

13

14

15

16

17

18

19

20

21

22

23 
Acute Mountain Sickness and Cognition

\section{Introduction}

3 Acute mountain sickness (AMS) commonly occurs following acute exposure to high

4 altitude (> 2,500m or $8200 \mathrm{ft}$ ) (Hackett \& Roach, 2012; Wilson \& Imray, 2009). AMS is

5 defined by symptoms including headache, dizziness or lightheadedness, nausea and

6 vomiting, difficulty sleeping, and fatigue. The Lake Louise self-reported score (LLS) is

7 one of the most widely used diagnostic scoring systems to diagnose AMS (Roach et al.,

8 1993).

10 Previous work has suggested the possibility of cognitive impairment (CI) in AMS. While

11 it is known high altitude conditions can impair cognition, and alter executive, memory

12 and language processes (Asmaro et al., 2013; Du et al., 1999; Kramer et al., 1993; Li et

13 al., 2000; McCarthy et al., 1995; Petrassi et al., 2012; Turner et al., 2015; Virues-Ortega

14 et al., 2004; Wu et al., 1998), it is generally accepted that altitude-related CI occurs

15 independently of AMS (Kramer et al., 1993; Virues-Ortega et al., 2004). However, some

16 studies do suggest CI and AMS are associated (Bian et al., 2015; Forster, 1985; Regard et

17 al., 1991, Shukitt-Hale et al., 1991, Issa et al., 2016). AMS is a symptom complex that

18 may be due to early, mild cerebral edema and increased intracranial pressure (Lawley et

19 al., 2016; Sagoo et al., 2016). Cerebral manifestations of acute altitude illness span from

20 common, benign AMS to potentially fatal high altitude cerebral edema (HACE). AMS

21 has no obvious neurologic signs (e.g., ataxia), but these are found in HACE. Because

22 high altitude cerebral illnesses appear to be a continuum from the mild symptoms of

23 AMS to severe symptoms with overt CNS findings of HACE, our hypothesis was that a 
Acute Mountain Sickness and Cognition

1 sensitive objective test for high altitude-related mild CI using an easily available field

2 technique would correlate closely with the presence and severity of AMS.

4 The Quick Mild Cognitive Impairment (Qmci) screen has potential to detect mild CI in

5 the field at high altitude. The Qmci screen rapidly and reliably detects early mild CI

6 (Molloy et al., 2005; O'Caoimh et al., 2012). We tested a slightly modified version of the

7 Qmci (which we refer to as the Environmental Quick Mild Cognitive Impairment Screen

8 (eQmci)) and sought to determine if AMS is associated with CI. We compared the eQmci

9 to the LLS and screened for medication by use of drugs for prophylaxis of AMS.

\section{Methods}

\section{Study Population}

13 All of our subjects had recently and rapidly ascended by vehicle and foot to the two

14 testing sites in the Annapurna region of the Nepalese Himalayas. Adult native English

15 speaking subjects visiting the Himalayan Rescue Association aid post in Manang at 3520

$16 \mathrm{~m}(11,550 \mathrm{ft})$ or at a temporary aid post in Thorong Phedi at $4550 \mathrm{~m}(14,930 \mathrm{ft})$ were

17 offered enrollment into the study. Surveys were only offered to clinically stable subjects

18 at the end of their medical visit after clearance from the health professional caring for

19 them. Inclusion criteria included native English-speaking adults between the ages of 18

20 and 65 years trekking through Manang or Thorong Phedi. This included asymptomatic

21 subjects who presented themselves to the clinic for education and prevention of illness.

22 Subjects were not excluded for having minor ailments including joint pain, blisters,

23 rashes, diarrhea and cough. Exclusion criteria included a history of dementia and 
Acute Mountain Sickness and Cognition

1 recreational drug or alcohol use. All recruited subjects were trekkers who had ascended

2 from $8000 \mathrm{ft}(2440 \mathrm{~m})$ within 1 week. No permanent residents of high altitude were

3 recruited.

4

5 Study Procedures

6 Subjects who presented to the aid posts meeting criteria were enrolled. A consent

7 document with information about the study was presented to each volunteer and written

8 consent was obtained. Information collected from each subject included age, years of

9 education, history of dementia, fluency in English, rate of ascent, medication use, and

10 self-rated high altitude experience. Subjects were asked about prophylactic and treatment

11 medications for high altitude illness, analgesics and any potentially sedating drugs during

12 their trek through the Annapurna region. Medications for general medical problems (e.g.

13 antihypertensives, oral contraceptives, thyroid replacement, etc.) were not recorded. Each

14 subject enrolled was administered the LLS and eQmci by a single investigator. This study

15 was approved (Protocol number 2014P001803) by the Partners Institutional Review

16 Board (Boston, MA, USA) and Nepali Health Research Council.

$18 \quad$ Testing

19 The LLS uses a 5-item scale, which surveys a subject's assessment of headache,

20 dizziness or lightheadedness, fatigue or weakness, gastrointestinal distress, and difficulty

21 sleeping. Each symptom is graded on a scale of 0 (not present) to 3 (severe). The

22 presence of a headache (score at least one for headache) and a total score of greater than

23 or equal to 3 is required to diagnose AMS (Roach et al., 1993). 
Acute Mountain Sickness and Cognition

2 The Qmci consists of 6 subtests including orientation, registration, clock drawing,

3 delayed recall, verbal fluency and logical memory. It has a median administration time of

44.24 minutes (O'Caoimh et al., 2013). The range of score is 0 to 100. Two modifications

5 were made to better apply this screen in high altitude wilderness settings. We removed

6 the clock drawing task given the practical concern of needing to remove gloves to

7 perform the test in a very cold environment. The clock drawing test is useful to assess

8 visuospatial cognition, but is clinically a less useful subtest when compared to other

9 elements in the Qmci (O'Caoimh et al., 2013). Instead, in order to maintain a distracting

10 task before the verbal recall exam, subjects were asked to count backwards from 100 by

11 sevens. A second modification was made in which proper names replaced common

12 names for repetition and recall testing. Proper names were matched for frequency of

13 occurrence in the English language. High altitude seems to have a more dramatic effect

14 on the recall of proper names while recall of common names is relatively resistant to

15 hypoxia (Pelamatti et al., 2003). The remaining subtests were unchanged. Subjects with

16 normal cognition have a median score of 75 (O'Caoimh R, 2014). Subjects who score $\leq$

1767 out of 100 meet criteria for mild CI and those who score $\leq 53$ out of 100 meet criteria

18 for dementia (O’Caoimh R, 2014).

20 Statistical Analysis

21 Continuous variables were compared using two-sample t-tests while categorical variables

22 were measured using frequency and percentage and compared using chi-square tests. The

23 relationship between the two measures was summarized using a Spearman correlation 
Acute Mountain Sickness and Cognition

1 coefficient. Two-sided $p$ values $\leq 0.05$ were considered as statistically significant. All

2 analyses were conducted using SAS version 9.4 (SAS Institute, Cary, NC).

4 Results

5 Seventy-nine subjects were enrolled in the study. All subjects who enrolled completed

6 the surveys. Twenty-two subjects (28\%) met criteria for AMS and 17 subjects (22\%)

7 met criteria for mild CI.

9 Table I presents demographic subject information stratified by presence of AMS and CI.

10 We found a significant difference in recent acetazolamide use between groups with and

11 without AMS. Twenty-three out of 79 (29\%) took acetazolamide in the last 24 hours. Of

12 these subjects, $10(43 \%)$ had AMS and 11 (48\%) had CI. A higher percentage of

13 subjects diagnosed with AMS had taken acetazolamide in the previous 24 hours

14 compared to those without AMS (45\% vs. $23 \%, \mathrm{p}=0.047)$. Similarly, a higher

15 percentage of subjects with CI had taken acetazolamide in the last 24 hours compared to

16 those without CI (65 \% vs. $19 \%, \mathrm{p}=0.0003)$. Subjects with CI were slightly older $(37 \pm$

1714 vs. $29 \pm 9, \mathrm{p}=0.058)$ and there were no other significant differences in group

18 characteristics [e.g. age, sex, nationality, education, days above $8000 \mathrm{ft}$, self-rated

19 experience at high altitude, and paracetamol and non-steroidal anti-inflammatory

20 (NSAID) use] in subjects with and without AMS, or with and without CI.

22 As this study tested subjects at two different altitudes, we also analyzed the results from

23 only Manang, where the majority of subjects were recruited $(n=62)$. When subjects from 
Acute Mountain Sickness and Cognition

1 Thorong Phedi were excluded, there was still a significant difference in recent

2 acetazolamide use between groups with and without AMS. Specifically, a higher

3 percentage of subjects diagnosed with AMS had taken acetazolamide in the last 24 hours

4 compared to those without AMS (44\% vs. $13 \%, \mathrm{p}=0.009)$. Similarly, a higher

5 percentage of subjects with CI had taken acetazolamide in the last 24 hours compared to

6 those without CI (54\% vs. $12 \%, \mathrm{p}=0.001)$. There were no other significant differences

7 in group characteristics.

9 There was a very weak correlation between the eQmci score and LLS based on the

10 Spearman correlation coefficient $(\mathrm{r}=-0.24, \mathrm{p}=0.04)$ as only $5 \%$ of the variance in the

11 eQmci can be attributed to LLS (see Figure 1). Among the 79 subjects, 6 (8\%) tested

12 positive for both AMS and CI and 46 (58 \%) tested negative for both. Sixteen subjects

13 (20\%) met the LLS criteria for AMS but had no CI. The remaining 11 subjects (14\%)

14 were cognitively impaired but did not have AMS. Those with concordance between the

15 eQmci and LLS were more likely to be male (69 \% vs. $41 \%, \mathrm{p}=0.014)$ and there was no

16 other significant difference in subject demographic characteristics between those with

17 and without concordance between the eQmci and LLS.

19 Components of the LLS were also analyzed as presented in Table II. There was no strong

20 correlation between the eQmci score and any of the LLS components, including

21 headache. The strongest correlation was seen in gastrointestinal symptoms, which was

22 still relatively weak $(\mathrm{r}=-0.28, \mathrm{p}=0.011)$. 
Acute Mountain Sickness and Cognition

1 We conducted a further analysis to examine the effect of acetazolamide on eQmci score.

2 Figure 2 shows the distribution of eQmci from those subjects with and without

3 acetazolamide use in the last 24 hours. The overall mean eQmci scores were lower in

4 subjects who took acetazolamide ( 69 vs. $77, \mathrm{p}=0.0007)$. The finding was consistent

5 when limited to subjects with the same LLS score. Table III compares the prevalence of

6 CI from subjects with and without acetazolamide use in the last 24 hours. Overall, CI

7 were more common among subjects with acetazolamide use $(47.8 \%$ vs. $10.7 \%, \mathrm{p}=$

8 0.0006). When stratified by AMS status, the finding remained consistent. Among those

9 without AMS, CI was significantly more frequent in subjects who took acetazolamide

10 than in subjects who had not taken the drug $(46.2 \%$ vs. $11.4 \%, p=0.011)$. Similarly,

11 among subjects with AMS, those who took acetazolamide showed a trend to have more

12 CI (50.0\% vs. $8.3 \%, \mathrm{p}=0.056)$ than those who had not taken the drug.

\section{Discussion}

15 Acute ascent to high altitude can impair CNS function including short-term memory,

16 working memory, and executive functioning (Asmaro et al., 2013; de Aquino Lemos et

17 al., 2012; Hornbein et al., 1989; Kennedy et al., 1989; Kramer et al., 1993; Petiet et al.,

18 1988; Petrassi et al., 2012; Shukitt-Hale et al., 1994; Turner et al., 2015; Virues-Ortega et

19 al., 2004). We hypothesized that a field test for mild CI would provide an objective

20 measure of altitude-related CNS dysfunction which would correlate with the presence

21 and severity of AMS. Our results do not suggest a significant correlation between the

22 eQmci and LLS to support this hypothesis. 
Acute Mountain Sickness and Cognition

1 Lack of relationship between cognitive impairment and acute mountain sickness

2 The lack of correlation between the LLS and eQmci suggests that CI occurs

3 independently of AMS. This is consistent with previous research (Virues-Ortega et al.,

4 2004). Krammer et al. (1993) tested twenty climbers at $4360 \mathrm{~m}(14,304 \mathrm{ft})$ ascending

5 Denali. While they demonstrated deficits in learning and retention in perceptual and

6 memory tasks, they did not correlate with AMS. In one study that tested mental capacity,

7 subjects with AMS were worse in pursuit aiming compared to subjects without AMS, but

8 in the remaining battery of cognitive tests, no significant differences were found (Bian et

9 al., 2015). It is possible that there is a difference in the aspects of cognitive deficits seen

10 with and without AMS. In the study by Regard et al. (1991), subjects simulated ascent to

$114500 \mathrm{~m}(14,734 \mathrm{ft})$ over 24 hours in a hypobaric chamber. Those with AMS had

12 deficiencies in short-term memory, but improved in conceptual tasks while those without

13 AMS had improved short-term memory, but no improvement in conceptual tasks (Regard 14 et al., 1991).

16 The lack of any relationship in our study and in others between CI and AMS, both of

17 which are inextricably linked to the hypoxemia of high altitude and are largely

18 neurological in their expression, is somewhat counterintuitive. However, hypoxemia can

19 very rapidly lead to cognitive deficits (usually without symptoms) in a span of minutes

20 even in healthy persons, whereas AMS develops more slowly and generally is not evident

21 for many hours. Furthermore, people differ widely in the risk for AMS as a result of

22 varying sensitivity to hypoxemia involving pathways not fully understood, but possibly

23 related to trigeminal nerve sensitivity and/or very mild brain swelling that may not 
Acute Mountain Sickness and Cognition

1 necessarily impair neuronal functioning. Thus CI at high altitude and AMS likely have

2 different pathophysiological bases, but may coexist in some people on any occasion.

4 Cognitive impairment at altitude

5 Our study found a surprisingly high prevalence of CI: 17 subjects (22\%) met criteria for

6 mild CI. In subjects with an age range similar to that in our study, no subject tested at

7 sea-level was found to have CI (Molloy, 2015). An incidental finding of our study was

8 that acetazolamide use, independent of the severity of AMS was associated with CI at

9 high altitude.

11 The discriminating power of the Qmci for detecting CI is influenced by age; specifically

12 it is less accurate for subjects older than 75 years (O'Caoimh, 2014). The cut-off of 67

13 used in this study is recommended for younger adults ( $\leq 75$ years) with more education

14 ( $\geq$ high school). All subjects in our study were in this category. At this cut-off the Qmci

15 has $86 \%$ sensitivity and $89 \%$ specificity for detecting the presence of mild CI. CI is not

16 seen in a young healthy educated population (Molloy, 2015). Sex does not significantly

17 affect cut-off scores (O'Caoimh R, 2014).

19 Acetazolamide and cognitive impairment

20 In subjects taking acetazolamide, we found a high incidence of both AMS and of CI.

21 Acetazolamide has been associated with confusion (Swenson, 2014). In a randomized

22 study of subjects at high altitude, subjects who received prophylactic acetazolamide (125

23 mg twice per day) had impaired neuropsychological measures of concentration, cognitive 
Acute Mountain Sickness and Cognition

1 processing speed, reaction time, short-term memory, and working memory (Wang et al.,

2 2013). In a similar study, subjects who took acetazolamide (500 mg slow release, once

3 per day) actually showed improvement in certain psychological tests (White, 1984). In

4 addition to higher daily acetazolamide dosing, this second study differed from the former

5 in that subjects traveled on foot rather than being airlifted to a higher altitude which may

6 be a confounding factor. A more recent study found that at sea level, acetazolamide use is

7 associated in a dose-dependent fashion with a spectrum of psychometric impairments,

8 including poor concentration, imbalance and slower reaction time responses (Collier,

9 2016). Our study did not differentiate between prophylactic or treatment doses, but a

10 future study can be designed to match groups with regard to this variable. Physiologically,

11 acetazolamide functions as a carbonic anhydrase (CA) inhibitor. CA plays a role in signal

12 processing, synaptic plasticity, memory, nerve conduction and cerebral oxygenation and

13 consumption (Brechue et al., 1997; Sun \& Alkon, 2002; Wang et al., 2015). CA

14 inhibition is likely responsible for the deficits in cognition. Whether CI arises from the

15 drug-induced metabolic acidosis that partially compensates for the magnitude of the

16 respiratory alkalosis (hypocapnia) or from more direct effects in the CNS is not easily

17 resolved, but a combination of the two may be likely. At sea level only $50 \%$ of patients

18 taking acetazolamide (250 mg, four times a day) have relief of common CNS side effects

19 with concurrent bicarbonate supplementation sufficient to correct the metabolic acidosis

20 (Lichter, 1981).

22 Limitations of the study 
Acute Mountain Sickness and Cognition

1 There are several limitations in this investigation. First, we made modifications to the

2 eQmci (the clock drawing task was replaced by reciting serial sevens and the repetition

3 and recall of names were changed from common to proper). Though the Qmci has been

4 validated against the Montreal Cognitive Assessment and Standardized Mini-Mental

5 State Exam, the two minor changes in the eQmci were not validated prior to testing. This

6 potentially could have an effect on the cut-off values originally tested for the Qmci.

7 Second, traveling to a distant, culturally unfamiliar location may alter one's performance

8 on a cognitive test. Third, there was a relatively small sample size. Other trends may be

9 been seen with a more robust sample. Unfortunately, our recruitment was limited as a

10 result of the devastating 2015 earthquake in Nepal, which resulted in premature

11 termination. Fourth, there may be several secondary influences on neuropsychological

12 function including fatigue, hypothermia, desynchronosis, upper respiratory infections,

13 and exposure to a new environment. An individual diagnosis was not recorded for every

14 patient. However, most presentations to the clinic that were unrelated to altitude were

15 quite minor (e.g. blisters) and would not be expected to affect cognitive function. Fifth,

16 there was no low-altitude control group, nor were our subjects tested at low altitude to

17 examine baseline scores. Sixth, there were no measurements of arterial oxygenation

18 saturation by pulse oximetry. In future studies, this would be a better indicator of cerebral

19 hypoxic stress than the actual altitude. Seventh, there were no longitudinal data to

20 determine if low eQmci scores correlate with the development of HACE. Defining the

21 clinical significance of mild CI and determining whether it can it be used to predict those

22 that will develop HACE or experience other clinical relevant sequelae are areas of future

23 research. In addition, there were no longitudinal data on the discontinuation of 
Acute Mountain Sickness and Cognition

1 acetazolamide. Eighth, subjects were studied at two different altitudes. However, the

2 results were not significantly changed when the data were limited to Manang, where most

3 subjects were tested. Ninth, in a convenience sample, in which subject self-selection

4 exists (researchers have no control or insight in subjects' decisions to continue to ascend

5 or not), unmeasured confounding variables may exist that could reduce the validity of our

6 conclusions.

$8 \quad$ Future Studies

9 Regardless of AMS, screening of mild CI may be useful for early detection of impaired

10 cognition and poor decisional judgment at high altitude. Our study, however, does not

11 demonstrate any clinical significance of having mild CI diagnosed by a cognitive

12 function test, since we did not measure rates of adverse health events or poor decision-

13 making, but this is a potential area of future research. Cognitive tests are sensitive and

14 have the potential to screen for hypoxic impairment at early stages before subjects

15 develop symptoms or demonstrate poor judgment (Stepanek et al., 2013). Other cognitive

16 tests have been employed at high altitude such as clock drawing (Quigley \& Zafren,

17 2016). The eQmci can be administered in less than five minutes, does not require any

18 special equipment, and has multiple versions available to minimize a learning effect

19 when administered to the same subject over different points in time (Cunje et al., 2007).

20 Future longitudinal studies in trekkers, as well as validation of the eQmci and comparison

21 to other cognitive tests, may be useful to investigate the clinical relevance of mild CI.

22 Further study of secondary influences on CI at high altitude (e.g. exercise, medications,

23 sleep quality) are also needed. Lastly, the comparison of CI with other objective 
1 measures (e.g., optic nerve sheath diameter, ultrasonography, cerebral magnetic

2 resonance imaging, serum biomarkers) might help advance a better understanding of

3 acute altitude-related CNS disease.

4

5 In summary, our data demonstrate only a weak correlation between the LLS and eQmci,

6 but a surprising prevalence of CI, an objective measure of CNS dysfunction, at high

7 altitude. CI was prevalent in subjects both with and without AMS. The presence of CI in

8 otherwise asymptomatic trekkers raises concerns for unrecognized CI at altitude.

9 Furthermore, we found that acetazolamide use, independent of the severity of AMS was

10 associated with CI at high altitude. Further research using CI testing may help define

11 objective measures of CNS dysfunction at altitude.

14 Acknowledgements: We are grateful to Dr. Willie Molloy, Dr. Ronan O’Caoimh,

15 Anthony Brohan, Dr. Marjorie Nicolas, Dr. Remi Drozd, Dr. Allison Mulcahy, and Emily

16 Ledingham for their contributions to this study.

\section{Table and Figure Legend}

21 Table I: Basic demographics. Percentages are reported with respect to different subgroups

22 (LLS <3, LLS $\geq 3$, eQmci > 67, eQmci $\leq 67)$. 
Acute Mountain Sickness and Cognition

1 Table II: Analysis of components of the LLS Associated with the eQmci score is

2 demonstrated with relatively weak correlation.

4 Table III: The relationship between eQmci scores and acetazolamide use were analyzed.

5 The overall mean eQmci scores were significantly lower in subjects that took

6 acetazolamide.

8 Figure 1: A scatter plot was created between LLS and eQmci scores. There was poor

9 correlation between the eQmci score and the LLS based on the Spearman (-0.24)

10 correlation coefficients.

12 Figure 2: A graphic display of the range of values for the eQmci in subjects with and

13 without acetazolamide use in the last 24 hours. eQmci scores were lower in subjects who

14 took acetazolamide $(\mathrm{p}<0.001)$.

19 References

Asmaro, D., Mayall, J., \& Ferguson, S. (2013). Cognition at altitude: impairment in executive and memory processes under hypoxic conditions. Aviat Space Environ Med, 84:1159-65. 
Brechue, W. F., Koceja, D. M., \& Stager, J. M. (1997). Acetazolamide reduces peripheral afferent transmission in humans. Muscle Nerve, 20(12), 1541-1548.

Cunje, A., Molloy, D. W., Standish, T. I., \& Lewis, D. L. (2007). Alternate forms of logical memory and verbal fluency tasks for repeated testing in early cognitive changes. Int Psychogeriatr, 19(1), 65-75.

Collier D. J., C. B. W., Anne-Marie Hedges, John Nathan, Rod J. Flower, James S. Milledge, and Erik R. Swenson. (2016). Benzolamide improves oxygenation and reduces acute mountain sickness during a high altitude trek and has fewer side effects than acetazolamide at sea level. Pharmacol Res Perspect, 4(3), doi: $10.1002 / \operatorname{prp} 2.203$.

de Aquino Lemos, V., Antunes, H. K., dos Santos, R. V., Lira, F. S., Tufik, S., \& de Mello, M. T. (2012). High altitude exposure impairs sleep patterns, mood, and cognitive functions. Psychophysiology, 49(9), 1298-1306.

Du, J. Y., Li, X. Y., Zhuang, Y., Wu, X. Y., \& Wang, T. (1999). Effects of acute mild and moderate hypoxia on human short memory. Space Med Medical Engineering, 12(4), 270-273.

Forster, P. J. (1985). Effect of different ascent profiles on performance at 4,200 m elevation. Aviat Space Environ Med, 56(8), 758-764.

Hackett, PH, Roach, RC, High-altitude medicine. In: Wilderness Medicine. P.A. Auerbach, Editor. 2012, Elsevier: New York, p. 1-35.

Hornbein, T. F., Townes, B. D., Schoene, R. B., Sutton, J. R., \& Houston, C. S. (1989). The cost to the central nervous system of climbing to extremely high altitude. $N$ Engl J Med, 321(25), 1714-1719.

Issa, A. N., Herman, N. M., Wentz, R. J., Taylor, B. J., Summerfield, D. C., \& Johnson, B. D. (2016). Association of Cognitive Performance with Time at Altitude, Sleep Quality, and Acute Mountain Sickness Symptoms. Wilderness Environ Med, 27(3), 371-378.

Kennedy, R. S., Dunlap, W. P., Banderet, L. E., Smith, M. G., \& Houston, C. S. (1989). Cognitive performance deficits in a simulated climb of Mount Everest: Operation Everest II. Aviat Space Environ Med, 60(2), 99-104.

Kramer, A. F., Coyne, J. T., \& Strayer, D. L. (1993). Cognitive function at high altitude. Hum Factors, 35(2), 329-344.

Lawley, J. S., Levine, B. D., Williams, M. A., Malm, J., Eklund, A., Polaner, D. M., . . . Roach, R. C. (2016). Cerebral spinal fluid dynamics: effect of hypoxia and implications for high-altitude illness. J Appl Physiol (1985), 120(2), 251-262.

Lichter, P. R. (1981). Reducing side effects of carbonic anhydrase inhibitors. Ophthalmol, 88(3), 266-269.

Li, X. Y., Wu, X. Y., Fu, C., Shen, X. F., Yang, C. B., \& Wu, Y. H. (2000). Effects of acute exposure to mild or moderate hypoxia on human psychomotor performance and visual-reaction time. Space Med Medical Engineering, 13(4), 235-239.

McCarthy, D., Corban, R., Legg, S., \& Faris, J. (1995). Effects of mild hypoxia on perceptual-motor performance: a signal-detection approach. Ergonomics, 38(10), 1979-1992.

Molloy, D. W., Standish, T. I., \& Lewis, D. L. (2005). Screening for mild cognitive impairment: comparing the SMMSE and the ABCS. Can J Psychiatry, 50(1), 5258 . 
Acute Mountain Sickness and Cognition

Molloy, D. W. (Oct 15th, 2015). personal communication.

O'Caoimh, R., Gao, Y., Gallagher, P. F., Eustace, J., McGlade, C., \& Molloy, D. W. (2013). Which part of the Quick mild cognitive impairment screen (Qmci) discriminates between normal cognition, mild cognitive impairment and dementia? Age Ageing, 42(3), 324-330.

O'Caoimh, R., Gao, Y., McGlade, C., Healy, L., Gallagher, P., Timmons, S., \& Molloy, D. W. (2012). Comparison of the quick mild cognitive impairment (Qmci) screen and the SMMSE in screening for mild cognitive impairment. Age Ageing, 41(5), 624-629.

O’Caoimh R., Gallagher P, Eustace J, Molloy W. . (2014). Cognitive Screening Tests Need to be Adjusted for Age and Education in Patients Presenting with Symptomatic Memory Loss. Irish J of Med Sci, 183 S(7) p314.

Pelamatti, G., Pascotto, M., \& Semenza, C. (2003). Verbal free recall in high altitude: proper names vs common names. Cortex, 39(1), 97-103.

Petiet, C. A., Townes, B. D., Brooks, R. J., \& Kramer, J. H. (1988). Neurobehavioral and psychosocial functioning of women exposed to high altitude in mountaineering. Percept Mot Skills, 67(2), 443-452.

Petrassi, F. A., Hodkinson, P. D., Walters, P. L., \& Gaydos, S. J. (2012). Hypoxic hypoxia at moderate altitudes: review of the state of the science. Aviat Space Environ Med, 83(10), 975-984.

Quigley, I., \& Zafren, K. (2016). Subtle cognitive dysfunction in resolving high altitude cerebral edema revealed by a clock drawing test. Wilderness Environ Med, 27(2), 256-258.

Regard, M., Landis, T., Casey, J., Maggiorini, M., Bartsch, P., \& Oelz, O. (1991). Cognitive changes at high altitude in healthy climbers and in climbers developing acute mountain sickness. Aviat Space Environ Med, 62(4), 291-295.

Roach R. C., Bartsch P., Hackett P. H., et al. The Lake Louise acute mountain sickness scoring system. In: Sutton JR, Houston CS, Coates G, editors. Hypoxia and Mountain Medicine 1993. Burlington, VT; Queen City Printers Inc. p. 272-4.

Sagoo, R. S., Hutchinson, C. E., Wright, A., Handford, C., Parsons, H., Sherwood, V., Wayte, S., Nagaraja, S., Ng'Andwe, E., Wilson, M. H. Imray, C. H. (2017). Magnetic Resonance investigation into the mechanisms involved in the development of high-altitude cerebral edema. J Cereb Blood Flow Metab, 37(1), 319-331.

Shukitt-Hale, B., Banderet, L. E., \& Lieberman, H. R. (1991). Relationships between symptoms, moods, performance, and acute mountain sickness at 4,700 meters. Aviat Space Environ Med, 62(9 Pt 1), 865-869.

Shukitt-Hale, B., Stillman, M. J., Welch, D. I., Levy, A., Devine, J. A., \& Lieberman, H. R. (1994). Hypobaric hypoxia impairs spatial memory in an elevation-dependent fashion. Behav Neural Biol, 62(3), 244-252.

Stepanek, J., Cocco, D., Pradhan, G. N., Smith, B. E., Bartlett, J., Studer, M., Kuhn, F., Cevette, M. J. (2013). Early detection of hypoxia-lnduced cognitive impairment using the King-Devick test. Aviat Space Environ Med, 84(10), 1017-1022.

Sun, M. K., \& Alkon, D. L. (2002). Carbonic anhydrase gating of attention: memory therapy and enhancement. Trends Pharmacolog Sci, 23(2), 83-89. 
1 Swenson, E. R. (2014). Safety of carbonic anhydrase inhibitors. Expert Opin Drug Saf, 2 13(4), 459-472.

3 Turner, C. E., Barker-Collo, S. L., Connell, C. J., \& Gant, N. (2015). Acute hypoxic gas

4 breathing severely impairs cognition and task learning in humans. Physiol Behav, $5 \quad 142,104-110$.

6 Virues-Ortega, J., Buela-Casal, G., Garrido, E., \& Alcazar, B. (2004).

$7 \quad$ Neuropsychological functioning associated with high-altitude exposure.

$8 \quad$ Neuropsychol Rev, 14(4), 197-224.

9 Wang, J., Ke, T., Zhang, X., Chen, Y., Liu, M., Chen, J., \& Luo, W. (2013). Effects of acetazolamide on cognitive performance during high-altitude exposure. Neurotoxicol Teratol, 35, 28-33.

Wang, K., Smith, Z. M., Buxton, R. B., Swenson, E. R., \& Dubowitz, D. J. (2015). Acetazolamide during acute hypoxia improves tissue oxygenation in the human brain. J Appl Physiol (1985), 119(12), 1494-1500.

White, A. J. (1984). Cognitive impairment of acute mountain sickness and acetazolamide. Aviat Space Environ Med, 55(7), 598-603.

17 Wilson MH, Imray CH (2009). The cerebral effects of ascent to high altitudes. Lancet Neurol., 8(8), 175.

Wu, X., Li, X., Han, L., Wang, T., \& Wei, Y. (1998). Effects of acute moderate hypoxia on human performance of arithmetic. Space Med Med Eng, 11(6), 391-395. 